\section{EL CONSTRUCTIVISMO SOCIAL EN LA CIENCIA Y LA TECNOLOGÍA: LAS CONSECUENCIAS NO PREVISTAS DE LA AMBIVALENCIA EPISTEMOLÓGICA*}

\author{
Ana Fernández Zubieta \\ SPRU-Science Policy Rearch Unit \\ University of Sussex
}

THE CONSTRUCTIVIST

APPROACH IN SCIENCE AND

TECHNOLOGY STUDIES: THE

UNINTENDED CONSEQUENCES

OF EPISTEMOLOGICAL

AMBIVALENCE

\begin{abstract}
The article summarises the main currents and contributions of constructivist approach in Science and Technology Studies. This approach portrays science as a collective enterprise. It shows that competing claims about nature indicate that techno-scientific evidence is flexible. The paper considers that epistemological agnosticism or atheism of the sociology of science to be a limitation in the constructivist approach. The paper notes that, paradoxically, in order to benefit from the insights of the constructivist approach an epistemological commitment is needed. This commitment requires admitting that it is possible and necessary to know what evidence is more robust, and what methodologies and theories are more powerful.
\end{abstract}

KEY WORDS: Social constructivism, epistemology, social epistemology, realist constructivism.

\section{INTRODUCCIÓN}

En ciencias sociales suele denominase construcción social al proceso por el cual una práctica que surge en una cultura o contexto social particular termina por parecer obvia o "natural» a aquellos que la practican. Desde este punto de vista, una preocupación esencial del constructivismo social como teoría sociológica del conocimiento consiste en descubrir las formas en que los grupos e individuos participan en la creación de la realidad social que perciben. El foco de atención se centra así en la manera en que los fenómenos sociales se crean, se institucionalizan y se convierten en realidades asumidas. Para el constructivismo, la realidad se construye socialmente en un proceso dinámico que se reproduce al actuar e interpretar el mundo. El constructivismo considera que "la realidad se construye socialmente y que la sociología del conocimiento debe analizar los
RESUMEN: El artículo presenta las principales corrientes y aportaciones del constructivismo social en la ciencia y la tecnología. Reconoce su contribución al ofrecer una imagen de la ciencia como una empresa colectiva y reivindicar una mayor flexibilidad de las interpretaciones y evidencias científico-tecnológicas. Para ello, señala que lo que caracteriza a estas corrientes es un ateismo o agnosticismo epistemológico. Indica que, paradójicamente, para aprovechar las aportaciones del constructivismo a la ciencia, y también a la ciencia social, es necesario adoptar un compromiso epistemológico que conlleva implicarse en un proceso en el que es preciso ofrecer criterios con los que sea posible reconocer qué evidencia es más robusta o qué métodos y teorias resultan más potentes.

PALABRAS CLAVE: Constructivismo social, epistemología, epistemología social, realismo constructivista.

procesos por los cuales esto se producen (Berger y Luckmann, 1967, 13).

Si la sociología de la ciencia se centra en un tipo de conocimiento específico, el conocimiento científico', su principal propósito se sitúa en analizar tanto el modo en el que los factores sociales influyen en la ciencia como la forma en que la ciencia influye en la sociedad. De esta doble relación, el análisis del modo en que los factores sociales intervienen en la ciencia se ha considerado problemático por sus posibles derivas relativistas. El relativismo sostiene que no existen criterios objetivos con los cuales validar los conocimientos científicos -que no existe la racionalidad y validez científica-. Sostiene que el contexto social, o los factores sociales, determinan los modos de observación, métodos y teorías científicas -contenido científico-. El problema del relativismo consiste, de este modo, en que 
encierra en sus enunciados su propia trampa. Es decir, si el conocimiento científico es relativo, los enunciados del relativismo, o de la sociología relativista, también lo son. $Y$, por lo tanto, carecen de objetividad y validez ${ }^{2}$. Un relativista contraargumentaría que esa falta de validez no es relevante.

La sociología de la ciencia mertoniana -institucional- surgió en un entorno en el que la sociología del conocimiento americana estaba definiendo, en un sentido similar, sus límites ante la llegada de la obra de Mannheim Ideología y Utopía (1936) ${ }^{3}$. Para evitar las derivas epistemológicas que conlleva considerar qué hace a la ciencia científica y abordar las implicaciones de admitir ciertos grados de influencia de los factores sociales en los criterios de validez científica, Robert Merton situó el ámbito de estudio de la sociología de la ciencia en el contexto en el que surge y se desarrolla la ciencia, alejándose del análisis de la validez de la ciencia (el contenido). Merton centró el análisis sociológico en la institucionalización de la ciencia ${ }^{4}$. Así, la sociología de la ciencia institucional de carácter clásico se alejó de las cuestiones epistemológicas, que quedaron como un terreno de análisis más propio de la filosofía de la ciencia.

En cambio, el constructivismo se ha definido en relación con el contenido de la ciencia -teorías y métodos- y en oposición al estudio del contexto científico -institucionesy al tipo de sociología que se encargaba de su estudio. El constructivismo pretende extender el análisis sociológico a la racionalidad científica para estudiar la influencia de los factores sociales en los procesos de validez y justificación científica. En sus propios términos, pretende abrir la «caja negra" (Whitley, 1972) de la ciencia al estudio sociológico. Para el constructivismo la propia sociología se había autolimitado al no abordar el estudio de los factores sociales en los procesos de racionalización de la ciencia ${ }^{5}$. El constructivismo en los estudios sociales sobre la ciencia, por tanto, intenta desentrelazar el modo en que los factores sociales entretejen el contenido de la ciencia ${ }^{6}$.

Las propuestas constructivistas han encontrado numerosas críticas en disciplinas como la filosofía de la ciencia o la sociología ${ }^{7}$. Sin embargo, dentro de las propias corrientes constructivitas también pueden encontrarse elementos críticos contra diversos alcances y derivaciones del su programa. Por ejemplo, Langdom Winner (1993) alerta de los peligros de centrar el análisis sociológico de la ciencia en el mero desvelar el carácter construido de la ciencia: "abrir cajas negras»". Este tipo de críticas son más comunes en la rama del constructivismo social más ligada a los estudios culturales de la ciencia que, con una propuesta de reivindicación más política y centrada en reivindicaciones identitarias (como el género, raza o el postcolonialismo), han denunciado las limitaciones de la Sociología del Conocimiento Científico (SCC). Lo que constituyen las propias cajas negras de la SCC (Grossberg et al., 1992). Es decir, cómo al centrarse en determinados aspectos sobre la validez de la ciencia han olvidado plantear cuestiones como el género, raza, etc. Por ello, es conveniente distinguir entre las Sociologías del Conocimiento Científico (SCC) (Sociology of Scientific Knowledge SSK) y los Estudios Sociales de la Ciencia y la Tecnología (ESCT) (Science Technology Studies: STS) posteriores que tienen un carácter menos radical en su sentido epistemológico en su propuesta. En este artículo se presta especial atención a las críticas «internas» del constructivismo con la pretensión de no dar una imagen sesgada y unilateral de este grupo de estudios.

El artículo recopila las aportaciones y autores fundamentales de las corrientes constructivistas. Presenta los puntos de unión de las diversas ramas así como las propuestas críticas. En el repaso de las críticas se presta una especial atención a las que han surgido del debate sobre la labor de los científicos como asesores en la toma de decisiones políticas y sobre el papel que el constructivismo puede desempeñar en su estudio. Por medio de ellas, se repasa la apuesta por el "realismo constructivista" y el problema de la agencia -capacidad de acción- a la hora de tomar decisiones y establecer compromisos sociales en los que intervenga la ciencia. El artículo finaliza recordando las aportaciones y críticas del constructivismo social y demandando la necesidad de un compromiso epistémico. Es decir, de reconocer la necesidad de toda actividad científica -también científico social- de ofrecer razones específicas -robustas o fuera del punto de vista de los actores- que, reconociendo sus limitaciones, permitan contribuir a la toma de decisiones colectivas.

\section{Corrientes constructivistas}

Dentro de las corrientes constructivistas en ciencias sociales se pueden incluir una gran variedad de enfoques y 
escuelas. En este apartado se llevará a cabo una presentación somera de las principales corrientes que pueden ser incluidas bajo esta etiqueta y de sus principales ideas y representantes. Entre ellas, el programa fuerte, la escuela de Bath, los estudios de laboratorio y aproximaciones micro, la teoría del actor-red, la teoría del conflicto y los estudios culturales de la ciencia.

\subsection{El programa fuerte}

El Ilamado programa fuerte fue la corriente que demandó más intensamente el giro constructivista para la sociología de la ciencia. Quizá por ello su propuesta alternativa está más ligada a una crítica a la sociología institucional mertoniana y su carga de referencias epistemológicas es más elevada. Surgió a principios de los setenta en el grupo interdisciplinar de la Science Unit de la Universidad de Edimburgo y agrupó a una serie de autores como David Bloor, Barry Barnes, R. G. A. Dolby o Donald MacKenzie. Knowledge and social Imaginary de Bloor (1976) recoge el manifiesto epistemológico y metodológico del grupo que se articula en torno a cuatro principios: causalidad, imparcialidad, simetría y reflexividad. Según esta corriente, la sociología debe explicar las causas de las creencias científicas -causal-, debe ser imparcial con respecto a la verdad o falsedad, racionalidad o irracionalidad o éxito -imparcial-, el mismo tipo de causas deben explicar los conocimientos verdaderos o falsos -simetría- y la misma sociología no puede escapar de sus propios patrones explicativos -reflexiva.

La mayor parte del sentido de estos principios residen en la reivindicación un terreno para el análisis sociológico que hasta entonces no había sido abordado por la sociología de la ciencia institucional. Según estos autores, la sociología institucional de la ciencia había seguido las limitaciones interpuestas fundamentalmente por la filosofía de la ciencia, ligada al estudio epistemológico y a una idea de racionalidad científica lineal y progresiva ${ }^{9}$. Así, sus reivindicaciones cobran especial sentido cuando se las interpreta en esta línea de reivindicación epistemológica para la sociología. Por ejemplo, uno de los principios más controvertidos, el principio de simetría, que revindica utilizar el mismo tipo de causas para explicar los conocimientos verdaderos y falsos, cobra mayor sentido si se lo interpreta como una defensa de mayor terreno explicativo para los factores sociales.
Hasta entonces en la explicación del contenido de la ciencia sólo se recurría a los factores sociales para explicar el conocimiento científicamente falso. Es decir, para explicar la frenología, el lysenkoísmo o el creacionismo una vez que se han aceptado como científicamente falsos o seudociencias. Así, los factores sociales permiten entender por qué ciertos conocimientos falsos son aceptados en determinadas circunstancias como científicamente verdaderos. El principio de simetría pretende alejarse de esta preconcepción que asocia el análisis de los factores sociales en la ciencia a lo falso, que la convierte en una seudodisciplina histórica ${ }^{10}$ y que había sido aceptada incluso por la propia sociología11. En este sentido el principio de simetría ha sido muy fructífero, aunque criticado incluso dentro de corrientes constructivistas [ej. el "Weak program» de Chubin y Restivo (1983)] por sus derivas relativistas ${ }^{12}$ y los inconvenientes añadidos a ella. En la misma línea, otros de los conceptos propuestos por el Programa Fuerte pueden resultar "ajenos" para otras corrientes constructivistas como "causalidad" o "reflexivismon (ej. Collins, 1983) por estar ligados al TRASD (Truth, Rationality, Success and Progress) -a lo que se interpreta como un entendimiento epistemológico del estudio de la ciencia- (Collins, 1981b).

Esta corriente considera que los intereses causan la acción social y el conocimiento científico. Para ello, revisan las controversias científicas analizando el papel que los intereses han desempeñado en ellas (Barnes, 1977; Barnes y Shapin, 1979; Barnes y Mackenzie, 1979). Por ejemplo, Barnes y Mackenzie (1979) dan cuenta de cómo la controversia estadística entre Pearson y Yule estaba condicionada por distintos intereses de clase. El primero más ligado con los intereses profesionales y los laboratorios del Colegio Universitario de Londres. El segundo más cercano a intereses aristócratas de la Royal Statistical Society. De este modo, muestran cómo algo exógeno como los intereses permea en el contenido de las disputas científicas y que éstas no se dirimen sólo por criterios científicos o racionales.

La escuela de Edimburgo se caracteriza por su enfoque macro y por aplicar una perspectiva histórica en sus análisis. Sin embargo, gran parte de las corrientes constructivistas posteriores han prestado más atención a cuestiones más micro y con métodos más etnográficos o de análisis de discurso.

ARBOR CLXXXV 738 julio-agosto [2009] 689-703 ISSN: 0210-1963

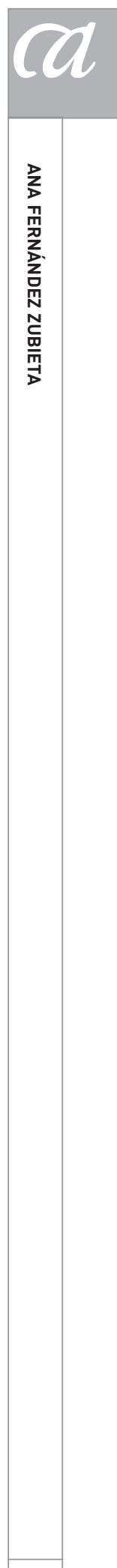

691 


\subsection{La escuela de Bath}

Para esta escuela las controversias científicas y los mecanismos de cierre de las mismas son el medio ideal en el que observar el proceso de construcción de la ciencia. $\mathrm{H}$. M. Collins ${ }^{13}$ se sitúa como representante más destacado de esta corriente al que se suman los trabajos de Pinch, Pickering, Travis y los posteriores de Bijker, Hughes, Pinch (1987) y el propio Collins sobre los desarrollos tecnológicos y el estudio de las viabilidades de las distintas alternativas técnicas. El programa relativista (Empirical Program of Relativism: EPOR) y su posterior aplicación al estudio de la técnica (Social Construction of Technology: SCOT) intenta mostrar la flexibilidad interpretativa de las decisiones científicas y técnicas, identificar los mecanismos sociales de cierre de esa flexibilidad y relacionarlos con un contexto social más amplio.

Para el programa relativista la naturaleza ofrece distintas posibilidades interpretativas que se dilucidan en las controversias científicas. El programa relativista debe dar cuenta de la flexibilidad interpretativa de los experimentos para sacar a la luz los mecanismos que operan en el cierre de controversias. Estos mecanismos de cierre no tienen que ver tanto con las evidencias científicas como con los propios procesos persuasivos que involucran a científicos y estructuras sociopolíticas más amplias ${ }^{14}$. Por medio del modelo de los siete pasos, Collins da cuenta del proceso en el que las percepciones y la inducción se van construyendo como algo que se considera exento de problemas. En las controversias la posición correcta sólo se configura una vez finalizada la disputa y no por la evidencia aportada sino por los propios mecanismos de cierre. No importa lo controvertidos que hayan sido los procesos de choque de opciones, una vez terminado el litigio, la alternativa triunfante adquiere carácter legítimo, evidente y científico por el mero hecho de ser la opción que ha terminado por imponerse. Las controversias son, en sentido kuhniano, una especie de momentos de creación microparadigmática.

Trevor Pinch y Wiebe Bijker ${ }^{15}$ (1987) en su extensión del programa relativista al análisis de la tecnología interpretan que uno de los factores que más limita la flexibilidad de la interpretación científico-tecnológica es el «grupo social relevante» y proponen como unidad básica de estudio el "core-set" o "núcleo humano", que incluye toda una serie de investigadores, becarios, administradores, proveedores, clientes, etc., y sus movimientos circunstanciales. En este sentido, recoge ideas que también se encuentran en la corriente del actor-red. Por ejemplo, cuando se analiza el diseño adoptado para la moderna bicicleta, se recoge el momento en el que encontraban disponibles distintos diseños y alternativas que terminaron estabilizándose en el "modelo actual». La construcción social de la tecnología asume que el diseño, significado y usos de la tecnología están infradeterminados por la naturaleza y son interpretativamente flexibles y, por tanto, no son autónomos. Es decir, no es el contenido de la ciencia o la virtudes tecnológicas lo que determina la adopción de una determinada tecnología sino una multitud de factores.

\subsection{Estudios de laboratorio y aproximaciones micro}

Esta corriente toma como objeto de estudio el medio científico por excelencia: el laboratorio. Alli, pretenden tener acceso a la "vida del laboratorio», a las prácticas reales de los científicos y no a lo que se reporta finalmente como resultado de su actividad. Los trabajos precursores de este enfoque son Laboratory Life de Bruno Latour y Steve Woolgar (1979) y The Manufacture of Knowledge de Karin Knorr-Cetina (1981), cuyo enfoque está más ligado a la corriente lingüista de Pierce ${ }^{16}$. Estos estudios declinaron en los noventa pero permanece su énfasis en el análisis de la ciencia como práctica (ej. Andrew Pickering).

Esta perspectiva considera que en la práctica de los laboratorios se genera el conocimiento científico de una forma muy diferente a la que transmiten los epistemólogos. El carácter que usualmente se adjudica a las investigaciones científicas (rigor, coherencia, etc.) es sólo el producto final de un proceso que recubre una serie de prácticas locales, contingentes y oportunistas. De este modo, la ciencia no funcionaría por medio de criterios universales preestablecidos, sino por prácticas circunstanciales. Para analizar estas prácticas, resulta imprescindible un estudio del lenguaje y la comunicación científica. El estudio de todo el proceso de la investigación permite concluir que los documentos científicos son sólo el punto culminante de un proceso de construcción en el que se van modulando las características con las que convencionalmente se relaciona el trabajo científico. La comunicación y la argumentación son claves en el proceso de persuasión en las distintas negociaciones que requiere la práctica científica. El estatus científico se va configurando mediante la omisión de las 
referencias a los agentes participantes, a sus acciones y a las circunstancias que rodean la acción.

Para este enfoque, la realidad no es la causa sino la consecuencia de los procesos de construcción del conocimiento. La distinción entre la naturaleza y la sociedad es el resultado de una serie de estrategias retóricas de argumentación y movilización de recursos que comienzan en el laboratorio pero que trasciende los límites del mismo ${ }^{17}$.

Dentro de este grupo se pueden incluir las propuestas etnometodológicas seguidoras de Garfinkel (1982), tales como Mullins (1973), Lynch (1985) o Garfinkel et al. (1981), donde se reivindica el carácter cotidiano y de sentido común de la actividad científica. Sostienen que uno de los indicios más claros del carácter construido de la ciencia se muestra en la limitación de los manuales de instrucciones y protocolos para fijar las conductas científicas. Las destrezas y habilidades de los científicos van más allá del método. Mulkay, Gilbert y Woolgar ${ }^{18}$ señalan especialmente la importancia del análisis del discurso científico para detallar las pautas discursivas que envuelven al trabajo científico.

\subsection{La Teoría del actor-red}

Las corrientes constructivistas son resultado de una serie de inquietudes disciplinares comunes. Por ello, resulta difícil seleccionar una corriente sin aludir a los rasgos que comparte con otras ${ }^{19}$. La idea básica de la teoría del actor-red, la idea de analizar unidades sociales más allá de organizaciones formales, puede encontrarse en autores como Restivo, Collins, en conceptos como el "core-set" del Epor/scot en los "mundos sociales» o, remontándonos a la sociología más institucional, en los colegios invisibles de Diana Crane. Sin embargo, su desarrollo se liga especialmente a la propuesta del École des Mines o Escuela de Paris de Latour y Callon, junto con John Law ${ }^{20}$.

La Teoría del actor-red surge a mediados de los años ochenta e intenta contrarrestar la tendencia microgeneralizada en los estudios sociales anteriores. Para ello, se pretende integrar los diversos niveles de la realidad social con una teoría que de cuenta de los distintos actores y factores que intervienen en la construcción de las redes de relaciones que permitan identificar los puntos de paso obligatorios. Es decir, situaciones indispensables que los actores han de admitir para poder cumplir con los intereses que le son asignados por su situación en la red.

Incorporan a su análisis el estudio de agentes y recursos en un intento por interconectar a la ciencia y la tecnología con fenómenos más clásicos como el poder. Para esta perspectiva es necesario estudiar la ciencia en acción (Latour, 1987); en su proceso de constitución, y antes de que la ciencia y la tecnología se conviertan en cajas negras. El proceso general incluye cuatro fases con diversas traducciones en las que los científicos intentan imponer su definición del problema al resto de fuerzas por medio de negociaciones que van desde la propia identidad de los distintos autores hasta sus posibilidades de interacción: problematización, interesamiento, enrolamiento y movilización.

La aportación más importante de estas corrientes la constituyen sus propios estudios como el de Pasteur (Latour, 1983), la expansión marítima portuguesa en los siglos XV y XVI de Law, o el de las vieiras en la bahía de St. Brieuc (Callon, 1986). Bajo el lema de "dame un laboratorio y moveré el mundo" analizan el carácter emprendedor de la ciencia. Quizá uno de los puntos más distintivos de la Teoría del actor-red es la fuerza de sus conceptos que obliga a pensar la actividad científica como algo intrínsecamente colectivo, dependiente de medio (actor-red) y no necesariamente humana (actante).

\subsection{La teoría del conflicto}

La perspectiva conflictivista en la ciencia tiene como mayores representantes a Randall Collins (1975) y Sal Restivo (1983). Es una corriente que no suele ser incluida en los estudios sobre las Sociologías del Conocimiento Científico (SCC) quizá por su origen americano que contrarresta con la predominancia europea de los SCC. Sitúa su objetivo en el análisis del poder en la ciencia y el rol del científico. Señalan cuatro tipos de roles para intelectuales y científicos: político, práctico, amenizador y educativo. La elaboración de Chubin y Restivo (1993) del weak program contra la neutralidad de la ciencia es un ejemplo de posturas críticas y de involucramiento de la ciencia social en el proceso de toma de decisiones que, posteriormente, generalizarán las corrientes culturales críticas de antropólogos y feministas.

Su propuesta quizá ha perdido relevancia por su aislamiento geográfico y temporal, ya que su enfoque pudo ser

ARBOR CLXXXV 738 julio-agosto [2009] 689-703 ISSN: 0210-1963

.


interpretado inicialmente como un acercamiento con un toque de crítica marxista un tanto extemporánea pero que posteriormente pudo resultar muy similar a las propuestas criticas de los estudios culturales americanos. Sin embargo, el análisis de Randal Collins ofrece una perspectiva sociológica muy interesante especialmente de la sociología de la sociología y filosofía y más en la línea de lo que pueden considerarse enfoques "neutros» 0 "no constructivos» de la ciencia -como la sociología institucional de la ciencia o la cienciometría.

\subsection{Los estudios culturales de la ciencia}

Este grupo de estudios también presentan divergencias de acuerdo a su procedencia más europea o americana. Los estudios americanos se centran más en las teorias del lenguaje de vinculación posestructuralista definidos en torno a cuestiones identitarias como el feminismo, sexualidad, raza o poscolonialismo ${ }^{21}$. La corrientes europeas pueden ligarse más con los debates sobre la ciencia y la sociedad de los años treinta y cuarenta y que fueron retomados a finales de los sesenta ${ }^{22}$.

Los estudios culturales de la ciencia, de inspiración frankfortiana, consideran que la ciencia ha participado en la "reificación» de los valores culturales categorizándolos como naturales. Por ejemplo, Dona Haraway (1991) ha denunciado cómo los científicos han ofrecido contenidos al sustento biológico de la idea de raza. Estos estudios incorporan análisis sobre el poder y la legitimidad de inspiración Foucaultiana. Es decir, de cómo la ciencia se ha puesto al servicio del poder en el desarrollo de unas tecnologías de poder que garantizan su ejercicio. También pueden encontrarse influencias de Bourdieu y su idea de capital simbólico en los estudios sobre reconocimiento y círculos de credibilidad. Esta influencia es más clara en el caso de Latour y Woolgar, aunque el propio Bourdieu se mantuvo critico con el enfoque de estos autores (1999).

Desde estos círculos se ha denunciado el carácter androcéntrico de la ciencia (Harding, 1986). Se estudia el modo en que la ciencia ha ignorado áreas de estudio y primado ciertos actores públicos restando "visibilidad" a otros actores y heterogeneidad a la sociedad. Con esta perspectiva reivindican la visibilidad de actores, enfermedades y puntos de vista que suelen quedar en un segundo plano del análisis científico.
Los movimientos ecologistas han dado lugar a otra fructífera vía de estudios de la ciencia y la tecnología. Bajo el lema "No en mi jardín» (Bullard, 1990) destapan las estrategias discursivas en los procesos de negociación donde se utiliza el lenguaje científico para aportar o restar credibilidad a una postura determinada. Los estudios sobre la resistencia a la tecnología o los efectos no queridos de la ciencia y la tecnología están mostrando la utilidad del enfoque constructivista para abordar cuestiones claves de las sociedades actuales en las que los retos científicos son más complicados (Stirling, 2001). Precisamente, esta vinculación del poder constructivista para dar cuenta de procesos científico-tecnológicos más ligados a cuestiones de cambio social más general potencia al enfoque constructivista al tiempo que incrementa sus demandas en torno a la necesidad de ofrecer alternativas. Así, su capacidad para analizar los factores sociales en el análisis de los riesgos científicos tecnológicos, en el entendimiento público de la ciencia, o foresight realza su capacidad para ampliar el espectro de posibilidades interpretativas en el proceso de toma de decisiones científico tecnológicas al tiempo que se pone a prueba su capacidad para dar respuestas.

\section{La acogida constructivista en España}

Dentro de España, ha abundado la revisión de los enfoques constructivistas de la ciencia, a pesar de la recepción tardía de las propuestas del Programa Fuerte (Beltrán, 1999). Según este autor, las primeras recepciones se producen a finales de los ochenta por parte de autores como Teresa González de la Fe ${ }^{23}$ y Jesús Sánchez Navarro (1988). En torno a esas mismas fechas, mediados de los 1985-1986, Esteban Medina (1989) organizó el curso de doctorado sobre el tema. De sus doctorandos, entre los que se encuentran Cristóbal Torres (1994), Juan Manuel Iranzo $(1995,1999)$, Alberto Cotillo y José Rubén Blanco, proceden las primeras traducciones de las corrientes constructivista para la ciencia, asi como aportaciones que resultan fundamentales para un acercamiento más exhaustivo que el que aquí se ha mostrado.

Dentro de las primeras traducciones y reflexiones sobre el enfoque constructivista cabe destacar el monográfico de Politica y Sociedad (14/15) de 1994 dedicado a sociología de la ciencia. Iranzo et al. (1995) recopila nuevas traduc- 
ciones y análisis por parte de autores nacionales de esta corriente. Iranzo y Blanco (1999) dedican un nuevo análisis de los inicios de esta corriente prestando mayor atención a los análisis del constructivismo aplicado a la tecnología. En la misma línea, Doménech y Tirado (1998) compilan nuevos ensayos sobre ciencia, tecnología y sociedad.

Dentro de los textos sobre ciencia y tecnología, cabe destacar Lamo de Espinosa et al. (1994), uno de los manuales más interesantes para acercarse al objeto y distintas tradiciones de sociología del conocimiento y de la ciencia ${ }^{24}$. En especial los capitulos de Cristóbal Torres sobre la tradición mertoniana y las sociologías del conocimiento científico resultan de especial relevancia para el tema abordado en este artículo. Más recientemente Torres ha recopilado las tradiciones intitutionalistas y también ha prestado atención al Entendimiento Público de la Ciencia (1995a) y a la ambivalencia y naturaleza dual de la tecnociencia (1995b). Sobre el entendimiento público y con una orientación más aplicada caben destacar los trabajos de Rafael Pardo y Félix Calvo (2002 y 2004). Otros trabajos en esta línea los ofrece Díaz de Rada et al. (1998). También se encuentran participaciones directas en el programa constructivista como la de Mikel Olazaran (1993) quien también ha reflexionado sobre la organización de los grupos de investigación y política científica (Olazaran et al., 2004) y los sistemas regionales de la ciencia.

Las sesiones organizadas en la Federación Española de Sociología sobre sociología del conocimiento y de la ciencia son un punto referente para los distintos autores españoles que analizan la ciencia y tecnología. En ellas se dan cabida a estudios empíricos y teóricos sobre la ciencia y la tecnología, en ellos las posiciones constructivistas han sido objeto de reflexión y guía teórica para estudios de caso. El capítulo sobre sociología del conocimiento, de la ciencia y la tecnología de Teresa González de la Fe, Cristóbal Torres Albero y Manuel Fernández Esquinas (2007) ofrece un panorama más completo de las aportaciones españolas en su aproximación al estudio de la ciencia.

La filosofía de la ciencia también ha revisado las aportaciones de la sociología constructivista. Aunque tienden a estar entroncados en una visión más filosófica y con tendencia más crítica, ofrecen argumentos fundamentales para situar el debate y las reacciones críticas al enfoque constructivista (ej. Solís, 1994, 1998). Marta González Gar- cía et al. (1997) ofrece un acercamiento filosófico positivo de esta corriente. Con un carácter más general merece la pena mencionar los trabajos de Javier Echeverría (1995 y 2003) y Fernando Broncano (ej. 2003 y 2006) que en sus obras ofrecen un gran espectro de cuestiones claves para entender el papel de la ciencia y la tecnología en la sociedad actual.

\section{La ambivalenCia del CONSTRUCTIVISMO SOCIAL Y SUS LIMITES}

A pesar de los elementos divergentes que pueden encontrarse dentro de la propia propuesta constructivista, las limitaciones de este enfoque se han visto acentuadas por lo que puede denominarse políticas de ESCT. Es decir, el debate del rol que el científico realiza como asesor en la toma de decisiones políticas y de las aportaciones que el enfoque del constructivismo social puede realizar al mismo. Como en todos los debates en los que se replantea la relación entre la ciencia y la sociedad, en él se mezcla una discusión entre la relación de la ciencia y la sociedad y las cuestiones epistemológicas.

Como se ha puesto de relieve, la propuesta constructivista parte de un cuestionamiento del contenido científico, de lo que se considera natural y certificado científicamente. En el origen del proyecto constructivista se encontraba la necesidad de reivindicar el poder explicativo de los factores sociales en la configuración del contenido científico. Todas las corrientes constructivistas parten de la asunción de que los factores sociales influyen en contenido científico. El problema se establece cuando se intenta precisar la extensión de la influencia de lo social en lo científico. Es decir, si no hay nada más que la mera construcción social -relativismo- o si queda algún papel a la evidencia científica. Esta cuestión ha sido desdeñada por las propias corrientes constructivistas por ser, ella misma, epistemológica (ej. Woolgar, 1988 y Latour, 1987).

El agnosticismo o ateísmo epistemológico (Zwanenberg y Millstone, 2000) no es una postura exclusiva del constructivismo. Otras corrientes, como el proyecto pragmatista de naturalización de la epistemología de Rorty (1979) o Quine (1969), sostienen que estudiar la ciencia a través de los criterios que la verifican acarrea más inconvenientes que

ARBOR CLXXXV 738 julio-agosto [2009] 689-703 ISSN: 0210-1963

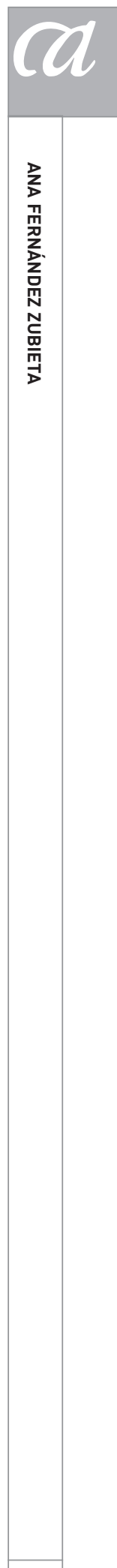

695 
ventajas. Conviene recordar que la salida del pragmatismo a la naturalización de la epistemología se produce con una apuesta por un compromiso político más fuerte. Es decir, utilizar criterios cívicos en lugar de epistemológicos. Sin embargo, la alternativa del constructivismo es el empirismo; empirismo que, por otro lado, ha resultado extremadamente productivo dando lugar a numerosos estudios de caso. Generalmente las corrientes constructivistas comparten el dictado de estudiar el contenido de la ciencia sin ninguna referencia epistemológica y con el empirismo como arma para evitar la epistemología.

\section{EMPIRISMO Y RECONSTRUCCIÓN: LA RESPUESTA DEL REALISMO CONSTRUCTIVISTA}

Los enfoques constructivistas proponen una estrategia deconstructiva como una manera de desligar los factores sociales, normativos (no-científicos) de las reivindicaciones sobre la naturaleza de los científicos. La estrategia deconstructiva consiste en encontrar contradicciones en la dicotomía social-natural que presenta el discurso científico. Esta estrategia tiene dos objetivos: el primero es antiepistemológico porque trata de desmontar las jerarquias "naturales" sobre las que se construyen las contradicciones. El segundo es encontrar la "voces secundarias" que se encuentran implícitas en una argumentación aparentemente univoca (Hess, 1997). Esta segunda estrategia es la que usan los estudios culturales y autores como Haraway (1991). Aunque no todos utilicen abiertamente la deconstrucción, todos comparte las razones que dan sentido a la estrategia deconstructiva: problematizar el discurso natural de la ciencia para romper la lógica epistemológica sobre la que está construido.

La estrategia deconstructiva es productiva porque "problematizan el rol de los científicos y cuestiona la dicotomía social-natural mostrando la flexibilidad de la interpretación de la evidencia científica. Autores como Wynne (1996) sostiene que la deconstrucción del discurso científico permite abordar las ultimas cuestiones que se encuentra encubiertas en el discurso científico. Defienden que el rol de los ESCT es abrir preguntas, problematizar agendas políticas, definiciones o el framing de asuntos de política pública establecidos (Singleton, 1998: 340). Según esta postura, este cuestionamiento abre la posibilidad de trascender el discurso desde unas visiones competitivas de la realidad a cuestiones sobre futuro. Sin embargo, no está claro que la estrategia constructiva conecte la primera intención de romper las dicotomías con la segunda de obtener beneficio de lo que se ha deconstruido.

Algunos autores critican la estrategia deconstructiva defendiendo que es necesario dar un paso adelante a la hora de transformar este rol "problematizador» de la metodología deconstructiva del constructivismo en una constructiva (Jasanoff, 1996: Radder, 1998; Zwanenberg y Millstone, 2000). El problema reside en que en ese paso deconstructivo no muestran la necesidad de reconstrucción ni ofrecen ningún criterio para llevarla a cabo. Problematizan el qué creer pero no ofrecen repuestas para el qué hacer (Radder, 1992, 1998). Por ejemplo, este autor reconoce la aportación del enfoque de Wynne sobre el efecto invernadero, pero lo critica por no ofrecer ninguna alternativa para abordarlo con una perspectiva diferente. Los críticos de la metodología deconstructiva muestran que los problemas del enfoque constructivista residen en que, en su negativa a abordar la cuestión epistemológica, no aportan una clave para la reconstrucción.

Como alternativa para hacer el constructivismo más productivo en el proceso de toma de decisiones políticas ofrecen la reivindicación del "realismo constructivista» (Jasanoff, 1996; Radder, 1998; Zwanenberg y Millstone, 2000, Chubin y Restivo, 1983). La defensa del realismo en el constructivismo implica reconocer que el mundo importa. 0 lo que es lo mismo, una vez mostrado que las distintas reivindicaciones sobre la naturaleza es un indicativo de que la evidencia científica es flexible, es posible y necesario saber qué evidencia es más robusta.

\section{El problema de la Agencia}

Otro problema fundamental del constructivismo tiene que ver con su concepción de la agencia (Hess, 1997; Amsterdamska, 1990). Este problema se observa claramente en la teoría del actor-red y los acercamientos reflexivos, pero también puede encontrarse en otras versiones de constructivismo como el análisis de los intereses de la escuela de Edimburgo (Barnes, 1977). El problema de imputación convierte a los científicos en meros «adictos al interés». Harry 
Collins (1983) trata de resolver esta cuestión reduciendo la labor de los intereses en el cierre de las controversias científicas y no en sus orígenes. En otras palabras, reduce el rol de los intereses con un análisis micro. La teoría del actor red trata de abordar este problema invirtiendo la cuestión y analizando los intereses como una consecuencia, más que una causa de la acción científica. Traslada la cuestión de las creencias de los científicos a la construcción de los hechos científicos. Para hacer esto, la teoría del actor-red establece la importancia de la acción no en las intenciones de los actores sino en las posiciones que los actores ocupan en las redes. Desde este punto de vista, la acción es resultado del poder de la red. Esta orientación ofrece a este enfoque la posibilidad de ampliar el espectro de la agencia a actores no-humanos o "actantes", porque la agencia no es una cuestión de intención sino más bien de atribución. Sin embargo, esta concepción más amplia de la agencia como una cuestión de atribución ha creado otros problemas para la contribución de la sociología de la ciencia al análisis de la política científica.

Si los actantes sólo orientan y dan sentido a sus acciones de acuerdo a sus posiciones en las redes, no hay lugar para la intencionalidad y responsabilidad de sus acciones. Los científicos sólo son replicantes del poder de la red y sólo buscan aumentar el poder de su posición en la red. La respuesta seria abrir este acercamiento a un enfoque más clásico del análisis del poder que atribuya a las acciones y significados de los actores más alternativas de las que provienen de las redes en la que se encuentra subsumidos.

Como reconocen Chubin y Restivo (1983), todas las tradiciones sociológicas tienen sus limitaciones porque son autosuficientes y desarrollan definiciones exclusivas de la ciencia y los investigadores. Sin embargo, los metaanálisis no son sólo necesarios para recuperar la necesidad de una asunción epistemológica sobre la naturaleza, también son necesarios por propósitos sociológicos.

\section{El COMPROMISO EPISTEMOLÓGICO}

Como se ha visto, los aciertos del constructivismo y su aportación al papel de la política de la ciencia están relacionados con su habilidad para cuestionar la autoridad epistemológica asumida del discurso científico. En ese sen- tido, este enfoque resulta muy fructifero para desenredar las diferentes (no científicas) fuentes de legitimación del discurso científico. Sin embargo, al tratar de hacer esto deconstruyendo la lógica epistemológica que esconde lo que "realmente" es una mezcla de argumentos científicos y no científicos del discurso natural de la ciencia, lo que consiguen es sólo cuestionar la autoridad epistemológica. La teoria del actor-red y las SCC sostienen que haciendo esto ayudan a cambiar las agendas políticas. Sin embargo, como sus críticos muestran, los constructivistas no cambian las cuestiones: sólo muestran que las cuestiones están mal planteadas. El reclamo constructivista referido a que están buscando nuevos actores, "voces secundarias" o cuestionando un aparente neutral discurso científico, sólo cuestiona la neutralidad de la ciencia. Sin embargo, no ofrece bases sobre las que explicar por qué esas voces secundarias y los actores que se encuentran detrás de ellas están excluidos. Es necesario reconocer el poder del enfoque constructivista para cuestionar visiones y actores en el discurso natural de la ciencia, pero también es necesario reconocer sus limitaciones para abordar cuestiones sobre cómo reformular los problemas y cómo incluir a los actores excluidos.

Las limitaciones del acercamiento constructivista provienen del mismo lugar que sus aciertos y precisamente éste puede ser el principal problema del programa. Como todos los acercamientos posmodernistas, resultan muy útiles a la hora de localizar contradicciones en teorias y discursos asumidos pero, en su negativa a ofrecer un criterio alternativo, se vuelven más dependientes de los criterios que quieren deconstruir. En otras palabras, la metodología deconstructiva de los programas constructivistas necesita un oponente que deconstruir. Como el adversario es la epistemología o las distinciones que emanan de su lógica, devienen más dependientes de los criterios epistemológicos. Es posible que la deconstrución sea un paso hacia futuras reconstrucciones como Wynne (1998) sugiere, pero también es ciento que puede ser un paso peligroso. Del mismo modo, el constructivismo necesita un criterio para detener el momento problematizador, para poder distinguir entre alternativas más robustas y para reconstruir lo que se ha deconstruido.

Como los defensores del realismo constructivista reconocen, se necesita un compromiso epistemológico para ser capaz de elegir entre distintas reivindicaciones del discurso 
natural. Es cierto que esta necesidad es más epistemológica que sociológica pero es sociológica -o sociológica en su vertiente crítica- si lo que se pretende es que el acercamiento constructivista sea más relevante en el análisis de la política científica. Como también es cierto que es sociológica si se reconoce la necesidad de buscar criterios fuera del punto de vista de los actores. Es decir, si se admite un compromiso epistemológico para la sociología. La propia sociología también necesita de externalidad si se admite que lo construido importa para la sociedad y no sólo contra la naturaleza. Como Bourdieu (2004) reconoce, decir que la ciencia es una actividad social, que las representaciones cientificas son representaciones sociales de la realidad no tiene demasiado sentido si no se especifican. Parafraseando a W. I. Thomas, es necesario reconocer que "cuando la gente define las situaciones como reales, son reales en sus consecuenciasn. Sin embargo y siguiendo a Marx, también hay que reconocer que la gente no define sus situaciones como quiere. Hay posiciones sociales desde las cuales es más fácil construir realidades sociales. Hay contextos científicos en los que es más fácil construir evidencia empírica.

Después de todo, cabe plantearse cuál es el sentido del debate epistemológico si parece terminar siempre encallado en una nueva versión de un debate antiguo. Quizá lo más relevante del debate epistemológico se sitúa en que, en definitiva, ayuda a definir o redefinir las relaciones entre la ciencia y la sociedad. En este sentido, la perspectiva constructivista permite plantearse la actividad científica como una empresa colectiva en la que se legitiman determinadas evidencias y afirmaciones sobre el mundo. Muestran que la evidencia tecnocientífica es más flexible $y$, de este modo, permite nuevas vías o actores que presenten otras alternativas. Esta visión de la ciencia resulta fructífera para establecer una descripción de la ciencia como una empresa colectiva, flexible y abierta. Ésta es una imagen de la ciencia que permite plantear instrumentos para abordar nuevos retos sociales, como son los problemas o riesgos globales en una sociedad cambiante. Sin embargo, para sacar partido de estas posibilidades que ofrece el constructivismo a la ciencia y la ciencia social, es necesario adoptar un compromiso epistemológico que implica involucrarse en un proceso en el que es necesario ofrecer criterios con los que sea posible reconocer qué evidencia es más robusta 0 qué métodos y teorías resultan más potentes. La sociología tiene que sea capaz de dar razones (epistémicas y no espistémicas) a la comunidad social a la hora de alcanzar un acuerdo. Esto implica reconocer que la racionalidad de la ciencia es una "racionalidad comunicativa" (Habermas, 1984) que tiene que ayudar a "hacer explícitas" (Brandom, 1994) las razones cuando hay un conflicto y no solamente un reporte descriptivo que trata de denunciar los aspectos sociales de la explicación científica. Implica reconocer que el poder explicativo de la sociología (epistemología social) no se incrementa contra la explicación "natural».

\section{Conclusión}

El repaso por las distintas corrientes de constructivismo social ha permitido dar una visión heterogénea de esta corriente. Reconoce que el constructivismo no implica asumir que no existen criterios de validez y justificación de la ciencia -relativismo- o implicarse en un debate estrictamente epistemológico. Sin embargo, admite que generalmente lo que caracteriza a esta corriente es un agnosticismo o ateísmo epistemológico que le lleva a desentenderse de ofrecer criterios alternativos de validez y justificación.

Este agnosticismo epistemológico proviene de su estrategia deconstructiva del discurso de validez científica, lo que le lleva a centrar su foco de atención en la ruptura de ese discurso. Como consecuencia, ofrecen una imagen más flexible de la evidencia científica. Al mismo tiempo, permiten identificar "voces secundarias" en el discurso científico, así como actores o problemas que permanecen relegados de la actividad científica. Pero, al mismo tiempo, ha provocado una actitud distante con respecto a la necesidad de ofrecer criterios de evidencia y validez sobre los que reconstruirla.

La perspectiva constructivista ha contribuido a ofrecer una imagen de la ciencia como una empresa colectiva en la que se legitiman ciertas evidencias y afirmaciones sobre el mundo. Muestran que la evidencia tecnocientífica es más flexible $y$, por lo tanto, puede estar abierta a nuevos caminos 0 actores que ofrezcan distintas alternativas.

Esta imagen de la ciencia resulta propicia no sólo para ofrecer una imagen de la ciencia más colectiva, flexible y abierta. Es una imagen de la ciencia que puede ofrecer 
herramientas para afrontar nuevos retos sociales como los problemas o riesgos globales en una sociedad cambiante. Sin embargo, indica que, paradójicamente, para aprovechar las aportaciones del constructivismo a la ciencia, y sobre todo a la ciencia social que estudia la actividad científica, es necesario adoptar un compromiso epistemológico que conlleva comprometerse en un proceso en el que es necesario ofrecer criterios con los que sea posible reconocer qué evidencia es más robusta o qué métodos y teorias resultan más potentes.

\section{NOTAS}

* La autora agradece los comentarios sobre versiones previas de este artículo de Erik Millstone, Andrew Stirling y Manuel Fernández Esquinas. Las limitaciones que permanecen en el texto son en su totalidad responsabilidad de la autora.

1 Establecer qué es lo que caracteriza al conocimiento científico es el principio del debate epistemológico. Si se articula en torno a uno criterios de validez del conocimiento científico, es necesaria una idea de racionalidad científica.

2 Sin embargo, admitir la influencia de los factores sociales en la ciencia, o el contenido científico, no equivale a decir que la determinen.

3 Para mayor detalle de la recepción de Mannheim en Estados Unidos ver Wolff (1967). Zubieta (2004) analiza la visión de Merton sobre Mannheim.

4 Robert Merton estudió las condiciones sociales que dieron lugar a la institucionalización de la ciencia en Ciencia, tecnología y sociedad en la Inglaterra del s.SVII, tesis que fue desarrollada entre 1933-35, y también los valores y normas de la ciencia en "A Note on Science and Democracy" (1942) - La estructura normativa de la ciencia-, para ocuparse del sistema de recompensas y comunicación de la ciencia posteriormente. The Sociology of Science (1973) recoge las principales aportaciones del autor.
5 El foco principal de sus críticas es la sociología de la ciencia mertoniana pero también reciben críticas los enfoques de Marx o Mannheim al considerar que las ciencia naturales y matemáticas tienen un estatus especial. Estas críticas son características de las primeras corrientes de SCC, en especial Bloor (1976).

6 Aunque ellos consideran que lo que hacen es establecer un acercamiento neutro sin tener en cuenta ningún a priori sobre la validez científica.

7 La mayor parte de estas críticas se centran en la carga relativista de esta corriente. Aunque conviene recordar que no hay nada en el constructivismo que implique una asunción del relativismo, uno puede admitir que los factores sociales influyen en el contenido de la ciencia sin afirmar que los determinan.

8 Denuncia el fallo de la Sociología del Conocimiento Científico (SCC) (Sociology of Scientific Knowledge SSK) en abordar los aspectos más politicos de la construcción científica. Este tipo de críticas considera que, al olvidar estos factores, se corre el peligro de "abrir cajas negras para encontrarlas vacias".

9 Para una discusión más detallada sobre la racionalidad científica y la dicotomía internalismo/externalismo construida sobre ella ver Shapin (1992), Medina (1983) o Solis (1994).

10 Seudociencia en el sentido de que necesita otra disciplina que establezca que algo es falso e histó-
Aceptado: 25 de septiembre de 2008 
rica porque interviene a posteriori, cuando se ha determinado que algo es falso.

11 Tanto Marx como Mannheim limitan el alcance explicativo de los factores sociales cuando se estudian las ciencias, en especial las matemáticas.

12 Si los mismos tipos de causas explican lo verdadero y lo falso, equivale a admitir que no existe lo verdadero.

13 Collins (1981 a y b) recoge la declaración programática y una serie de estudios en su edición del monográfico 11 de Social Studies of Science.

14 Estrategia que trasluce la influencia del segundo Wittgenstein, la fenomenologiia de Schutz y Mary Hesse.

15 Otras aportaciones importantes son MacKenzie (1990), Law (1987) Huhges (1987), Callon (1987).

16 Otros trabajos destacados son Law y R. J. Williams (1982); M. Zenzen y S. Restivo (1982). Woolgar (1988), Latour (1993) Knorr-Cetina \& Mulkay (1983) ofrecen los fundamentos básicos del enfoque.

17 Knorr-Cetina (1981) critica a Woolgar y Latour por haber relegado las relaciones sociales y cognitivas que trascienden del ámbito del laboratorio y que incluyen agencias de financiación, privadas y públicas, editores de revistas, etc. Toda una serie de relaciones sociales transepistémicas.

18 Entre ellas se encuentran los trabajos de G.N. Gilbert y M. Mulkay $(1981,1984)$ y Mulkay, J. Potter y S. Yearley (1983).

19 En otro artículo de este monográfico (Echeverría y González) se hace un tratamiento más extenseo de la teoría del actor-red. Por ello, este apartado se dedica a resaltar brevemente algunos de los rasgos de esta teoría en relación con la corriente constructivista.

20 Latour (1983), Law (1986) recoge unos de los estudios más conocidos de este enfoque. Ver el capitulo dedicado a este enfoque que incluye este monográfico para un acercamiento más detallado de este programa.

21 Grossberg (1992) ofrece una descripción de estos estudios

22 En los cuarenta la discusión enfrentaba a los "nuevos humanistas científicos" encabezados por John D. Bernal y la Cambridge Scientist' Anti-War Group con M. Polanyi de la Society for Freedom in Science. Los del primer grupo -Bernal, J. Crowther, Lancelot Hogben, Joseph Needham, Hyman Levy, J. B. S. Haldane, Farrington, Pledge- estaban influidos por Boris Hessen y su trabajo sobre los Principia de Newton para el Segundo Congreso Internacional de Historia de la ciencia, celebrado en Londres en 1931. Para más ínformación ver Werskey 1978, Graham, 1985, Ben-David, 1991 o Fernández Zubieta (2004).

23 También fundadora de la Asociación Española de Ciencia y Tecnología.

24 El trabajo de este autor sobre la reflexibidad (1990) ofrece un punto de vista relevante para un análisis de carácter más general sobre la teoría sociológica.

\section{BIBLIOGRAFÍA}

Amsterdamska, Olga (1990): "Surely You Are Joking, Monsieur Latour!", Science, Technology, \& Human Values, 25, 4, 495-504.
Barnes, Barry (1977): Interests and the Growth of Knowledge, London, Routledge.

Barnes, Barry y Mackenzie, Donald (1979): "On the role of interests in scientific change", en Roy Wallis (ed.), On the margins of science, Sociological Review Monograph 27, Keele, Staffordshire, University of Keele.

Barnes, Barry y Shapin, Steve (1979): Natural order, Beverly Hills, Sage.

Beltrán, Manuel (1999): "Prólogo", en Iranzo, Juan M. y Blanco, J. Rubén, Sociología del conocimiento científico, CIS/UP de Navarra, Madrid.

Ben-David, Joseph (1991): Scientific Growth. Essays on the Social Organization and Ethos of Sciencie, Berkeley, University of California Press.

Berger, Peter y Luckmann, Thomas (1968): The Social Construction of Reality, New York, Anchor.

Bijker, Wiebe; Hughes, Thomas P. y Pinch, Trevor (1987): The Social Construction of Technological Systems: New Directions in the Sociology and History of Technology, Cambridge Mass., The MIT Press.

Bloor, David (1976): Knowledge and Social Imagery, London, Routledge and Kegan Paul.

Bourdieu, Pierre (1999): Razones prácticas, Madrid, Anagrama.

- (2004): Science of Science and Reflexivity, Cambridge, Policy Press.

Brandom, Robert (1994): Making It Explicit: Reasoning, Representing and Discursive Commitment, Cambridge Ma. Harvard.

Broncano, Fernando (2003): Saber en condiciones, Madrid, A. Machado Libros.

- (2006): Entre ingenieros y ciudadanos. Filosofía de la técnica para días de democracia, Madrid, Montesinos.

Bullard, Robert (1990): Dumping in dixie: race, class, and environmental equito, Boulder, Westview. 
Callon, Michael (1986): "Some Elements of a Sociology of Translation: Domestication of the Scallops and the Fishermen", Power, Action and Belief: A New Sociology of Knowledge?, 32, ed. John Law, London, Sociological Review Monograph, Routledge and Kegan Paul.

- (1987): "Society in the making: The study of technology as a tool for sociological analysis", en Bijker, Huges y Pinch (eds.), Cambridge Mass., The MIT Press, 83-103.

Chubin, Daryl y Restivo, Sal (1983): "The 'Mooting' of Science Studies: Research Programmes and Science Policy", en Knorr-Cetina \& Mulkay (eds.), Science Observed, London, Sage, 53-83.

Collins, Harry (1981a): "Knowledge and Controversy: Studies of Modern Natural Science", Social Studies of Science, 11.

- (1981b): "What Is TRASP? The Radical Programme As a Methodological Imperate", Philosophy of the Social Sciences, 11, 215-24.

- (1983): "An Empirical Relativist Programme in the Sociology of Scientific Knowledge", en Knorr-Cetina \& Mulkay (eds.), Science Observed, London, Sage, 255-285.

Collins, Randall (1975): Conflict Sociology, New York, Academic Press.

Díaz de Rada, Vidal; Ayerdi, Peio y Olazaran, Mikel (1998): "Percepción social de la ciencia y la tecnología en España", Revista Internacional de Sociología, 21, 73-100.

Doménech, Miquel y Tirado, F. J. (comps.) (1998): Sociología simétrica. Ensayos sobre ciencia, tecnología y sociedad Barcelona, Gedisa.

Echeverría, Javier (1995): Filosofía de la ciencia, Madrid, Akal.

- (2003): La revolución tecnocientífica Madrid, FCE.

Fernández Zubieta, Ana (2004): Génesis y desarrollo interdisciplinar del progra- ma mertoniano para la ciencia, tesis doctoral, Madrid, Carlos III.

Garfinkel, Harold (1982): A Manual for the Study of Naturally Organized Ordinary Activities, London, Routledge \& Kegan Paul.

Garfinkel, Harold; Lynch, Michael y Livingston, Eric (1981): "The Work of a Discovering Science Construed With Materials From the Optically Discovered Pulsar", Philosophy of Social Sciences, 11, 131-58.

González de la Fe, Teresa y Sánchez Navarro, Jesús (1988): "Las sociologías del conocimiento científico", REIS, 43, 75-124.

González de la Fe, Teresa; Torres Albero, Cristóbal y Fernández Esquinas, Manuel (2007): "Sociología del conocimiento, de la ciencia y de la tecnología", en Manuel Pérez Yruela (coord.), La sociología en España. Madrid: CIS, 541-564.

González García, Marta I.; López Cerezo, José A. y Luján López, José L. (ed.) (1997): Ciencia, tecnología y sociedad, Barcelona, Ariel.

Graham, Loren R. (1985): "The Socio-Political Roots of Boris Hessen: Soviet Marxism and the History of Science", Social Studies of Science, 15, 705-22.

Grossberg, Lawrence; Nelson, Cary y Treichler (ed.) (1992): Cultural Studies, New York, Routledge.

Habermas, Jurgen (1984): The Theory of Communicative Action, Boston Ma., Beacon Press.

Haraway, Dona (1991): Simians, Cyborgs and Women: The Reinvention of $\mathrm{Na}-$ ture, New York, Routledge.

Harding, Sandra (1986): The science question in feminism, Itaca, Cornell University Press.

Hess, David (1997): Science Studies, New York, New York University Press.

Hill, Stephen; Bellavista, Joan; De Miguel Rodríguez, Jesús Manuel y Turpin,
Tim (1998): "Cultura organizativa de investigadores y entorno político y social", Revista de sociología, 54, 79109.

Hughes, Thomas (1987): "The evolution of large technological systems", en Wiebe Bijker, Thomas Hughes y Trevor Pinch (eds.), The Social Construction of Technological Systems, Cambridge, MIT Press.

Iranzo, Juan M.; Blanco, Rubén; González de la Fe, Teresa; Torres, Cristóbal y Cotillo, Alberto (comp.) (1995): Sociología de la ciencia y la Tecnología, CSIC, Madrid.

Iranzo, Juan M. y Blanco, Rubén (1999): Sociología del conocimiento cientifico, CIS/UP de Navarra, Madrid.

Jasanoff, Sheila (1996): "Beyond Epistemology: Relativism and Engagement in the Politics of Science", Social Studies of Science, 26, 2, 393-418.

Knorr-Cetina, Karin D. (1981): The Manufacture of Knowledge, Oxford, Pergamon Press.

Knorr-Cetina, Karin D. y Mulkay, Michael (1983): Science Observed, Beverly Hills, Sage.

Lamo de Espinosa, Emilio (1990): La sociedad reflexiva. sujeto y objeto del conocimiento sociológico, Madrid, CIS.

Lamo de Espinosa, Emilio; González García, José M. y Torres Albero, Cristóbal (1994): La sociología del conocimiento y de la ciencia, Madrid, Alianza.

Latour, Bruno (1983): "Give Me a Laboratory and I Will Raise the World", Science Observed, Karin D. Knorr-Cetina y Michael Mulkay (eds.), London, Sage.

- (1987): Science in Action, Cambridge, Harvard University Press.

Latour, Bruno y Woolgar, Steve (1979): Laboratory Life: The Social Construction of Scientific Facts, London, Sage.

Law, John (ed.) (1986): Power, action and belief, Sociological Review Monograph, London, Routledge. 
Law, John (1987): "Technology and heterogeneous engineering", en Wiebe Bijker, Thomas Hughes y Trevor Pinch (eds.), The Social Construction of Technological Systems, Cambridge, MIT Press.

Law, John y Williams, R. J. (1982): "Putting facts together: A study of scientific persuasion", Social Studies of Science, $12,535-58$.

Lynch, Michael (1985): Art \& artifact in laboratory science, Londres, Routledge \& Kegan Paul.

MacKenzie, Donald (1990): Inventing accuracy. A historical sociology of nuclear missile guidance systems, Cambridge Mass., The MIT Press.

Mannheim, Karl (1936): Ideology and Utopia: An Introduction to the Sociology of Knowledge, London, Kegan Paul.

Medina, Esteban (1983): "La polémica internalismo/externalismo en la historia y sociología de la ciencia", Reis, 23, 53-75.

- (1989): Conocimiento y sociología de la ciencia, Madrid, CIS.

Merton, Robert (1942): "A Note on Science and Democracy", Journal of Legal and Political Sociology, I, 115-26.

- (1973): The Sociology of Science: Theoretical and Empirical Investigations, Norman Storer (ed.), Chicago, Chicago University Press.

Mulkay, Michael (1981): "Action and belief or scientific discourse? A possible way of ending intellectual vasallage in Social Studies of Science", Philosophy of Social Science, 11, 163-171.

- (1984): "The scientist talks back: A one-act play, with a moral, about replication in science and reflexivity in sociology", Social Studies of Science, 14, 265-282.

Mullins, Nicholas (1973): "The development of specialities in social Science: the case of ethnomethodology", Social Studies of Science, 3, 245-73.
Olazaran, Mikel (1993): "A Sociological History of the Neural Network Controversy", Advancens in Computers, 37, 335-425.

Olazaran, Mikel; Lavía Martines, Cristina y Otero, Beatriz (2004): "¿Hacia una segunda transición en la ciencia?: política científica grupos de investigación", RES, 4, 143-172.

Pardo, Rafael y Calvo, Félix (2002): "Attiutdes towar Science among the European Public: a methodológical análisis", Public Understanding of Science, 11, 155-195.

- (2004): "The cognitive dimension of Public Perceptions of Science: Methodological Issues", Public Understanding of Science, 11, 155-195.

Pinch, Trevor y Bijker, Wiebe (1987): "The social construction of facts and artefacts: or how sociology of science and sociology of technology might benefit each other", en Wiebe Bijker, Thomas Hughes y Trevor Pinch (eds:), The Social Construction of Technological Systems, Cambridge, MIT Press.

Quine, W. V. (1969): Ontological Relativity and Other Essays, New York, Columbia.

Radder, H. (1992): "Normative Reflexions on Constructivist Approaches to Science and Technology", Social Studies of Science, 22, 1, 141-173.

- (1998): "The Politics of STS", Social Studies of Science, 28, 2, 325-331.

Restivo, Sal (1983): "The Myth of the kuhnian revolution", en Randal Collins (ed.); Sociology Theory, San Francisco, Jossey-Bass.

Rorty, Richard (1979): Philosophy and the Mirror of Nature, Princeton, Princeton U. Press.

Shapin, Seteve (1992): "Discipline and Bounding: The History and Sociology of Science As Seen Through the Externalism-Internalism Debate", History of Science, 30, 333-69.
Singleton, Vicky (1998): "The Politic(ian)s of SSK", Social Studies of Science, 28, 2, 332-338.

Solis Santos, Carlos (1994): Razones e Intereses, Barcelona, Paidós.

- (comp.) (1998): Alta Tensión: historia, filosofía y sociología de la ciencia. Ensayos en memoria de Thomas Kuhn, Barcelona, Paidós.

Stirling, Andrew (2001): "Participatory processes and scientific expertise: precaution, diversity and transparency in the governance of risk", Participatory Learning and Action, 40, 66-71.

Torres, Cristóbal (1994): Sociología política de la ciencia, CIS/Siglo XXI, Madrid.

- (2005a): "Representaciones sociales de la ciencia y la tecnología", REIS, 111, 9-43.

- (2005b): "La ambivalencia ante la ciencia y la tecnología", RIS 42, 938.

Werskey, Gary (1978): "Para comprender a Needham", en Joseph Needham, Ciencia, religión y socialismo, Barcelona, Crítica, 11-29.

Whitley, Richard (1972): "Black Boxism and the Sociology of Science: A discussion of the Major developments in the field", Sociological Review Monograph: The Sociology of Science, 18, 61-92.

Winner, Langdom (1993): "Upon opening the black box and finding it empty: social constructivism and the philosophy of technology", Science, Technology and Values, 18, 362-78.

Wolff, Kurt (1967): "The Sociology of Knowledge in the United States of America", Current Sociology 15 (1).

Woolgar, Steve (1988): Knowledge and Reflexivity. New Frontiers in the Sociology of Knowledge, London, Sage.

Wynne, Brian (1996): "SKK's Identity Parade: Signing-Up, Off-and-On", Social Studies of Science, 26, 2, 357-391. 
- $\quad$ (1998): "Reply to Radder", Social Studies of Science, 28, 2, 338-344.

Zenzen, Michael y Restivo, Sal (1982): "The mysterious morphology of immiscible liquids: A study of scientific practice", Social Science Information, 21, 447-73. Zwanenberg, P. y Millstone, E. (2000): "Beyond Skeptical Relativism: Eva- luating the Social Constructions of Expert Risk Assessments", Science, Technology, \& Human Values, 25, 3, 259-282. 